\title{
Analysis of the scalability of hierarchical IEEE 802.15.4/Zigbee networks
}

\author{
E. Casilari
}

\author{
A. Flórez-Lara \\ University of Málaga \\ ETSIT, Campus de Teatinos, 29071 \\ Málaga (Spain), Tel.: 34-952132755 \\ ecasilari@uma.es
}

J.M. Cano-García

\begin{abstract}
IEEE 802.15.4/Zigbee standard constitutes a promising technology for the deployment of low-power and low rate Wireless Personal Area Networks. Many practical applications of this type of networks may require the association of a nonnegligible number of nodes with strong power constraints. In that case, the most efficient way (especially in terms of battery consumption) of organising 802.15.4/Zigbee networks is by means of hierarchical cluster-trees. However, the standard does not impose any particular algorithm to organize, dimension or synchronize a cluster-tree network. This paper addresses this issue by proposing and comparing three simple strategies to parametrize the timing of superframes in static Zigbee clustertrees.
\end{abstract}

\section{Keywords}

Zigbee, IEEE 802.15.4, cluster-tree, beacon mode, sensor networks.

\section{INTRODUCTION}

The standards IEEE 802.15.4 (which specifies the Physical Layer and Medium Access Control [1]) and Zigbee [2] jointly describe a protocol stack for the definition of Wireless Personal Area Networks (WPAN). This stack is targeted to provide networking solutions for low-cost wireless embedded devices with consumption and bandwidth limitations. In particular the basic framework of IEEE 802.15.4 defines 10-meter communications with a transfer rate of $250 \mathrm{kbps}$, but this parameters can be decreased even more (down to $20 \mathrm{Kbps}$ in the 868/915 $\mathrm{MHz}$ band) to enable a lower power consumption in the Zigbee nodes. IEEE 802.15.4-compliant transceivers, which operate in the Industrial, Scientific and Medical (ISM) radio bands (including $2.4 \mathrm{GHz}$ band), are designed to be simpler and more economical (below 1 dollar) than the modules from other alternative standards for WPANs (such as Bluetooth). Besides, the standard also contemplates the possibility of real-time guarantees so it can be also applied in scenarios where real-time flows (e.g.: voice or the

Permission to make digital or hard copies of all or part of this work for personal or classroom use is granted without fee provided that copies are not made or distributed for profit or commercial advantage and that copies bear this notice and the full citation on the first page. To copy otherwise, or republish, to post on servers or to redistribute to lists, requires prior specific permission and/or a fee.

INFOSCALE 2008, June 4-6, Vico Equense, Italy

Copyright $\odot 2008$ 978-963-9799-28-8

DOI 10.4108/ICST.INFOSCALE2008.3490 signal from a biosensor) are expected to be transmitted. As a consequence, in spite of the immature state of Zigbee technology, it has become an appealing candidate to support a wide set of services, particularly for low consume domotic sensor networks (but also for other applications ranging from medical telemonitoring to industrial plant-process control.

The main attractiveness and also the main challenge of IEEE 802.15.4/Zigbee is its potentiality to set up self-organizing networks capable of adapting to diverse topologies, node connectivity and traffic conditions. In fact, most advantages of employing IEEE 802.15.4 strongly depend on the configuration of the Medium Access Control (MAC) sublayer.

The MAC layer of IEEE 802.15.4 enables two alternative operational modes: (1) under the non beacon-enabled or point-topoint mode, the access control is governed by non-slotted CSMA/CA, (2) under the beacon enabled mode, a coordinator node periodically sends beacons to define and synchronize a WPAN formed by several nodes. Networks operating without beacons permit nodes to transmit in any moment so they are highly scalable (they roughly behave as low rate 802.11 networks). However, this mode forces the nodes to be continuously listening to the wireless medium, which involves a low power efficiency (particularly if traffic load is not high). Additionally this technique does not allow nodes to reserve resources so real-time constraints of certain services cannot be satisfied. Conversely, in beacon-enabled networks nodes can stay inactive (in a low consumption state) most of the time and are only required to wake up just in time to receive the beacon from their coordinator and to keep synchronized with the network. Although the role of the nodes under the beacon mode is very asymmetrical, even the coordinator is not required to be active during the whole period between two consecutive beacons (Beacon Interval) and may enter in a power saving mode. This clearly diminishes the duty cycle of all the devices and enlarges the battery lifetime. In addition, the beacon mode enables nodes to reserve time slots (the so called Guaranteed Time Slots) within the Beacon Interval, which eases the transmission of real time traffic. As its main disadvantage, beaconing networks demand specific algorithms to coordinate and design the different parameters that govern the timing of the beacon process. If this issue may be of importance when just one single Zigbee tree (some nodes synchronized with a single coordinator) is considered, it is crucial when more complex network topologies are created by the association of several coordinators. In this sense, an inadequate parametrization of the beaconing technique may seriously impact on the network capacity. 
This paper studies the configuration of static hierarchical IEEE 802.15.4/Zigbee networks. In particular, the paper proposes and compares several algorithms to dimension the activity periods of the coordinators in a cluster-tree Network. The performed simulations and the corresponding analysis show the strong influence of this dimensioning on the network scalability.

This paper is organized as follows: Section 2 briefly describes the way in which beacon IEEE 802.15.4/Zigbee networks are configured. The section also reviews the existing strategies to avoid direct beacon collision in networks where multiple coordinators coexist. Section 3 presents three algorithms of increasing complexity to design this type of networks while Section 4 compares them by means of simulation. The final Section 5 summarizes the main conclusions and offers some possible research lines.

\section{CONFIGURATION OF 802.15.4/Zigbee NETWORKS}

IEEE 802.15.4 standard distinguishes two classes of nodes: the so-called Full-Function Devices (FFD) and the Reduced-Function Devices (RFD). FFDs are enabled to perform as network 'coordinators'. In that case, FFDs are in charge of the communications of a set (or 'cluster') of nodes (the 'children' nodes) following a star topology. On the other hand the role of RFD (which is reserved for very simple devices with limited resources) just permits the communication (as 'end' nodes) with just one FFD acting as its coordinator. In order to announce its presence (identifying the corresponding WPAN) as well to make possible the synchronization and the configuration of the children nodes, a coordinator may follow the beaconed mode. So the coordinator has to broadcast a special frame (a beacon) periodically. The time between two consecutive beacons of a coordinator is called the Beacon Interval $(B I)$. The BI is divided in an active part and an inactive part. Along the active part the coordinator set ups a Superframe of 16 equally-spaced time slots. All the transmissions to/from the coordinator and its children must take place during the superframe, whose duration is called the Superframe Duration $(S D)$, while in the inactive period of the $\mathrm{BI}$ all nodes (including the coordinator) may enter a power saving mode to extend the lifetime of their batteries. In that case all the devices would wake up just in the moment that the beacon is expected to be received.

The durations of the Beacon Interval and the Superframe are defined by means of two order parameters: the Beacon Order $(B O)$ and the Superframe Order $(S O)$, in the way:

$$
B I=a \cdot 2^{B O} ; \quad S D=a \cdot 2^{S O}
$$

being $a$ the Base Superframe duration (15.36, 24 or $48 \mathrm{~ms}$ depending if a bit rate of 250,40 or $20 \mathrm{kbps}$ is employed, respectively). The value of $B O$ is limited to the interval $[0,14]$ while the value of the $S O$ must be necessarily equal or lower than $B O$. In the case that $S O$ coincides with $B O$, the Superframe would extend along the whole Beacon Interval and no inactive period would exist. On the contrary, if $S O$ is set to a low value (compared to that of $B O$ ), the sleep or inactive period would occupy most of the beacon interval, resulting in an important reduction of the duty-cycles and the power consumption of the devices (this is obviously achieved at the cost of decreasing the network capacity or throughput).

As it is reflected in Figure 1 (which represents the structure of the IEEE 802.15.4 beacon interval), the superframe can in turn be divided in two parts: during the initial slots (Contention Access Period or CAP), the communications are governed by slotted CSMA/CA. Consequently, during the CAP, nodes compete to utilize the slots of the superframe. Although nodes listen the medium before transmitting, if two nodes initiate their emissions simultaneously collisions will occur. Collisions, which provoke the underutilization of the CAP slots, induce delay or even data losses if retransmissions fail after applying the typical backoff algorithm of CSMA. During the second part of the superframe (Contention Free Period or CFP), up to seven slots (Guaranteed Time Slots) can be directly reserved to particular children nodes to guarantee the quality of service of time sensitive applications.

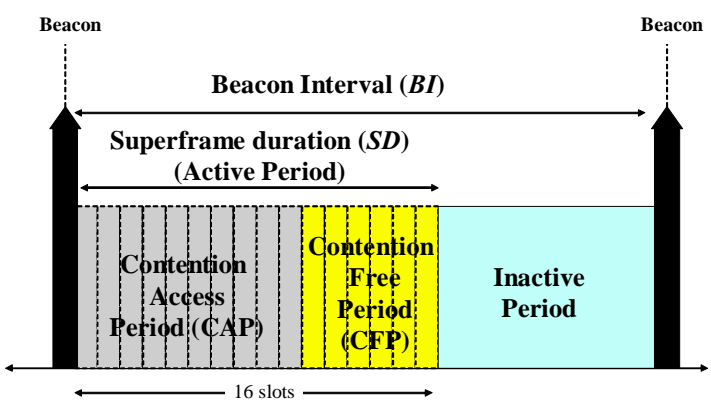

Figure 1. Structure of a 802.15.4. Superframe

\subsection{Management of beacons in clustered networks}

Apart from the configuration of tree networks with a single coordinator, the Zigbee standard contemplates the arbitrary association of cluster coordinators to form cluster-trees. Under this scheme, the cluster coordinators are also responsible for retransmitting the data from any 'child' node within their clusters when they desire to send data to any external node. One of the coordinator nodes configuring the whole cluster-tree network must assume the central role of the WPAN or Zigbee Coordinator (ZC). The rest of the coordinators are called the Zigbee Routers (ZR).

The Zigbee specification just suggests the concept of cluster-trees and does not impose any protocol nor algorithm to create and organize this type of networks. In fact, existing commercial Zigbee/802.15.4-compliants modules do not support the formation of cluster-tree topologies (some of them such as MICAz and TelosB [3] motes just implement the Physical Layer of 802.15.4). Thus, in the ambit of Wireless PAN, the organization of Zigbee cluster-trees constitutes a challenging issue that is still far from being solved.

In this sense, the most important problem of the coexistence of more than one coordinator is the possibility that beacons get lost due to collisions. Two beacons collide if they are simultaneously emitted by two adjacent coordinators (both in the same transmission area of each other). Even when the coordinators 
cannot intercommunicate directly, a child node can lose the beacon from its coordinator if its reception is interfered by the transmission of the other neighbor coordinator (effect of the hidden node).

In both cases the beacon collision provokes that children nodes get desynchronized from the coordinator and consequently prevent the correct operation of the network.

As long as IEEE 802.15.4/Zigbee standard does not address the problem of beacon collision, IEEE 802.15.4 Task Group 15.4.b [4] has proposed several generic strategies to cope with it [5]:

A method to avoid the direct collision of beacons introduces the so called beacon-only period, consisting in a time window that is specifically reserved for the transmission of all the beacons in the network. As it is illustrated in Figure 2, during this time window beacons are sequenced and emitted in a contention-free way so collisions cannot occur. After the beacon only period, the superframes of all the coordinator start at the same time. Making so, as it can be observed in the figure, the superframe duration of each cluster can be designed with independence of the rest. However the coexistence of active periods of different clusters augments the possibility of packet collision while it prevents the implementation of Guaranteed Time Slots. An algorithm to allocate and to schedule slots to the beacons during the beacon only period is also required to apply this policy, which alters the superframe structure defined in the standard.

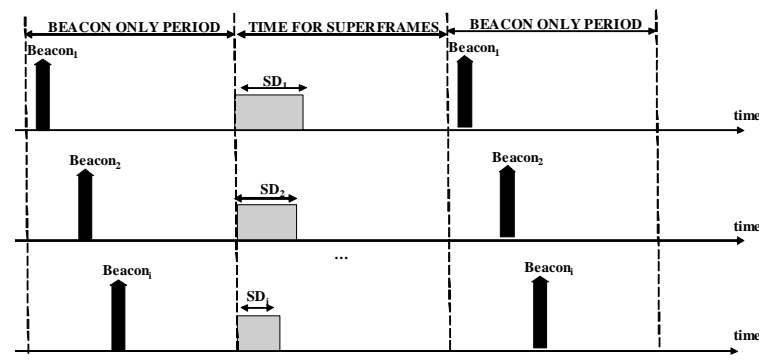

Figure 2. Collision avoidance by defining a beacon-onlyperiod

An alternative method to this previous mechanism proposes to arrange the active part of the superframes of neighbor coordinators so that they not overlap in the time. As it is illustrated in Figure 3, the idea now is to fragment the Beacon Intervals in such a way that the beacon and the corresponding superframe of a coordinator do not coincide with those of any other cluster. For this purpose, as it is indicated in the figure, all the superframes must be conveniently shifted a certain offset time.

This policy, which does not imply any change in the IEEE 802.15.4/Zigbee standard, enables the utilization of Guaranteed Time Slots and reduces the number of nodes contending in the Contention Access Period of any superframe to those belonging to a single cluster. However, the scheduling of beacons within the different Beacon Intervals and especially the duration of the superframes must be carefully designed. Otherwise, as far as the activity of a cluster obliges the neighbor clusters to stay inactive (for example, two coordinators cannot directly communicate except if one is the child of the other), the network could present serious scalability problems. Next section addresses this issue.

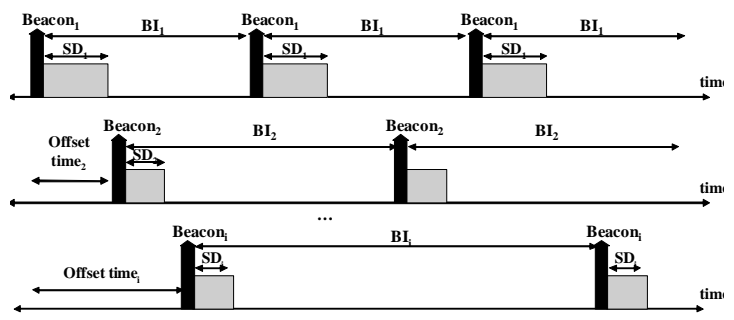

Figure 3. Collision avoidance by distributing the beacons and the superframes

\section{ALGORITHMS FOR DIMENSIONING THE SUPERFRAME ORDER}

Following the time division approach previously explained, in this section we propose several simple mechanisms to organize the timing of the superframes of the different clusters of a Zigbee network.

The general idea is that the utilization of the Beacon Intervals should be maximized to avoid as much as possible those periods in which no cluster is active and consequently the whole network remains idle. Just in the case that the expected traffic of the network is known to be extremely low (so that the offered throughput of the cluster-tree will not be a restriction) other policy should be considered to restrict the node activity.

In our analysis we assume the most pessimistic situation in which all the coordinators are in the transmission range of the rest (which can occur in an actual scenario if the deployment area of the IEEE 802.15.4/Zigbee network is physically reduced). In that case, any coordinator can interfere the rest, so the emission of a beacon cannot take place during the superframe of any other coordinator. This implies that the Beacon Intervals must be organized so that when one cluster is active the rest must be in the sleep (inactive) mode.

On the other hand, the Beacon Interval is straightforwardly related to the delay in the network as long as it determines the timing of the communication of a node and its coordinator. Thus, a node desiring to send a packet may have to wait at least the $B I$ until it receives the beacon of its coordinator. In a multihop network (such as the cluster tree) where the packets may traverse several coordinators (waiting for the corresponding beacons), this delay is accumulated from hop to hop so it can reach a noticeable value. In [6] a method is presented to adjust the Beacon Order to the traffic load but it is only valid for Zigbee networks with a simple star topology (the Superframe Order order of the unique network coordinator is merely fixed to a minimum).

The simplest way to impose an upper bound to this delay is to fix the same global Beacon Interval in all the nodes (by setting the parameter BO to the same value). In that case, the organization of the hierarchical Zigbee cluster-tree basically entails the definition of the duration of the Superframes of all the coordinators 
(including the Zigbee Coordinator). These durations are characterized by their corresponding Superframe Orders. The offset times between the beacons of the coordinators must be designed to distribute all the superframes during the BI without any overlapping. To guarantee this, the aggregation of all the Superframes must not be greater than the global Beacon Interval (BI):

$$
B I=a \cdot 2^{B O} \geq \sum_{i=1}^{N_{C}} S D_{i}=\sum_{i=1}^{N_{C}} a \cdot 2^{S O_{i}}
$$

where $N_{C}$ is the number of coordinators in the network (being $i=1$ the index for the Zigbee Coordinator, and $i=2$ to $N_{C}$ the indexes for the Zigbee Routers), while $S D_{i}$ and $S O_{i}$ are the Superframe Duration and Superframe Order of the $i$-th node, respectively.

The following sub-sections propose diverse policies to perform the election of the Superframe Orders in a generic hierarchical Zigbee cluster tree. The policies can be applied to static networks in which the number of coordinators and/or the topology are previously known.

\subsection{Equidistribution of the Beacon Interval}

As a first attempt to distribute the Beacon Interval among the coordinators, we set the Superframe Order of all nodes to the same value:

$$
S O_{i}=S O \quad \forall i \in\left[1, N_{C}\right]
$$

Taking into account the restriction of eq. (2) we have:

$$
a \cdot 2^{B O} \geq \sum_{i=1}^{N_{C}} a \cdot 2^{S O}=a \cdot N_{C} \cdot 2^{S O}
$$

From this inequation we can easily calculate the highest possible value that can be assigned to $S O$ :

$$
S O=\left\lfloor\log _{2}\left(\frac{2^{B O}}{N_{C}}\right)\right\rfloor=B O-\log _{2}\left(N_{C}\right)
$$

where the operator $\lfloor x\rfloor$ indicates the highest integer lower than $x$ (as long as the value of $S O$ must consist in a positive integer).

\subsection{Prioritization of the Zigbee Coordinator}

Most practical applications of Zigbee/802.15.4 technology consist of sensor networks in which the data collected in the nodes are forwarded to a gateway or central node which normally resides in the Zigbee Coordinator. The centralization of the flows in a hierarchical Zigbee network may provoke the ZC to become a traffic bottleneck. To avoid this situation, the previous policy in which all the nodes have the same Superframe Duration should be modified. A extremely simple modification to prioritize the role of the $\mathrm{ZC}$ is to design its Superframe Order $\left(\mathrm{SO}_{1}\right)$ with twice the value of that of the rest of the cluster coordinators (which in turn are designed to be equal), in the way:

$$
\begin{aligned}
& S O_{i}=S O \quad \forall i \in\left[2, N_{C}\right] \\
& S O_{1}=2 \cdot S O
\end{aligned}
$$

Substituting this parameters in the equation (2) we obtain that:

$$
a \cdot 2^{B O} \geq \sum_{i=1}^{N_{C}} a \cdot 2^{S O_{i}}=\underbrace{a \cdot 2^{2 \cdot S O}}_{S D_{1}}+\underbrace{a \cdot\left(N_{C}-1\right) \cdot 2^{S O}}_{\sum_{i=2}^{N_{C}} S D i}
$$

From where we can deduce the following quadratic inequation:

$$
\left(2^{S O}\right)^{2}+\left(N_{C}-1\right) \cdot 2^{S O}-2^{B O} \leq 0
$$

From the positive root of this inequation we can obtain again the maximum values of the Superframe Orders that guarantee that Beacons do not collide:

$$
S O=\left\lfloor\log _{2}\left(1-N_{C}+\sqrt{\left(N_{C}+1\right)^{2}+4}\right)-1\right\rfloor
$$

\subsection{Topology based distribution}

Even in the cases where all the network traffic is uplinked to the Zigbee coordinator, the previous method to design the SO values can imply a too rigid solution as it can give an excessive weight to the role of the Zigbee Coordinator. In many network topologies the nodes (and the traffic) can be asymmetrically distributed among the different clusters so that there can exist Zigbee routers that support even the same traffic load than the central Zigbee coordinator.

In this paper we propose a more flexible approach by which the Superframe Order of a coordinator is designed as a function of the traffic that the node is expected to support. As the global sum of the superframe duration is limited by the Beacon Interval the assignation must be accomplished in an iterative manner so that the superframe order of the nodes with a higher traffic load can be prioritized.

The particular proposed algorithm is schematized in Figure 4 and it proceeds as it follows.

1) All the Superframe Orders $\left(\mathrm{SO}_{i}\right.$, where $\mathrm{SO}_{1}$ corresponds to that of the Zigbee coordinator) are initially set to 0 .

2) If equation (2) is not satisfied, the network cannot be dimensioned without removing any cluster or increasing the present Beacon Interval (and consequently the packet delay).

If the sum of the Superframe Durations does not exceed the Beacon Interval:

3) Let $l_{i}$ be the number of leaf nodes 'depending' of the $i$-th coordinator. A node is said to be a 'leaf' if it generates uplink traffic. A leaf node depends on a coordinator if the coordinator is in the path from the leaf node to the Zigbee Coordinator. Accordingly, this coordinator will forward to the Zigbee Coordinator all the possible traffic flowing from the leaf node.

4) Increase in one unit the Superframe Order $\left(\mathrm{SO}_{j}\right)$ of the coordinator with the highest value of the associated parameter $l_{i}$. (In the first iteration this coordinator must necessarily be the 
Zigbee Coordinator). Using $l_{j}$ (related to the number of leaf nodes) as the weight to decide the value of $S O_{i}$, we implicitly presume that all leaf nodes will inject a similar traffic (which can be the case of many sensor networks). If the traffic generation is not expected to be balanced along the nodes, the algorithm could be adapted just setting the initial value of $l_{i}$ to the expected global traffic load of the corresponding $i$-th coordinator.

In the algorithm

5) If equation (2) can be fulfilled with this new value of $S O_{j}$, the corresponding value of $l_{j}$ is divided by 2 and the process returns to previous step (4).

6) On the contrary, if equation (2) cannot be satisfied, $\mathrm{SO}_{j}$ cannot be augmented without surpassing the Beacon Interval. As a consequence, $S \mathrm{SO}_{\mathrm{j}}$ must be assigned to its original value (decreased in one unit) and $l_{j}$ is set to 0 so that this coordinator cannot be selected if step (4) is executed again.

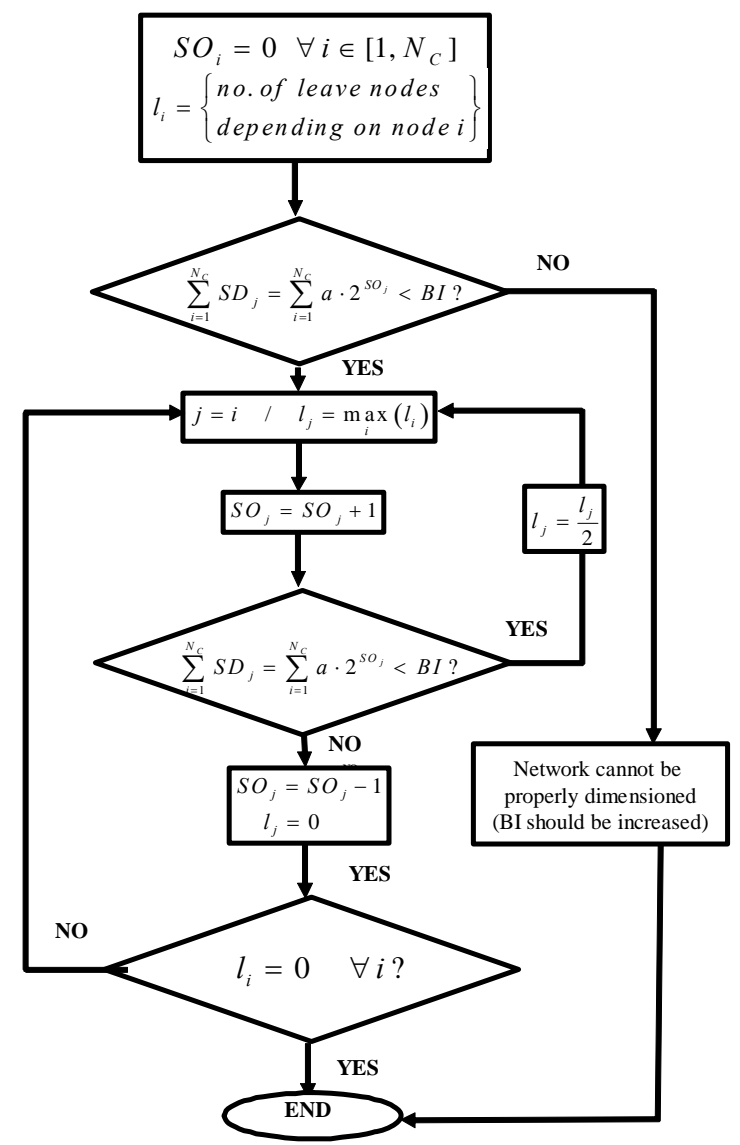

Figure 4. Proposed topology based algorithm for the dimensioning of the Superframe Order of the coordinators

7) If all the coordinators have a value of the parameter $l_{i}$ equal to zero, no SO can be increased and the Beacon Interval is supposed to have been completely distributed among the coordinators. In other case, return to step (4).

In contrast with other interesting proposals for the coordination of Beacon emissions in cluster-tree network [7], our algorithm permits to particularize the Superframe Order for each coordinator, so it can be adapted to different topologies and traffic loads.

\section{SIMULATION AND RESULTS}

To evaluate the performance of the precedent algorithms, we perform a set of simulations. The employed simulating software tool was specifically designed for this purpose on $\mathrm{C}++$. The packet level results of the simulations were formatted so they can also be analyzed with the Chipcon CC2420 Packet Sniffer [8].

We simulated three different network topologies depicted in Figures 5, 6 and 7. In our topologies, we consider a three-layer hierarchy in which leaf nodes (those generating traffic) do not have any children. This could correspond to a realistic scenario of a sensor network in which leaf nodes (the sensors) consist of simple RFD end devices while coordinators could be more complex mains powered FFD nodes. In the tests, no guaranteed time slots are employed in any cluster. The network configuration phase is neither considered in the analysis.

We simulated the performance of the algorithms under diverse traffic conditions. For comparison purpose, we describe the input traffic in the network by means of the concept of traffic load in the most loaded network cluster. More precisely, we define the traffic load $(\rho)$ as the ratio of the bytes that the leaf nodes try to inject and the maximum number of bytes that could be hypothetically transmitted at the Zigbee peak rate (250 kbps) during the superframe of the most critical cluster (i.e., the cluster that is supporting the highest data rate during the active period). In particular, $\rho$ is defined as a percentage in the way:

$$
\rho=\max _{i}\left(100 \cdot \frac{l_{i} \cdot n_{B I} \cdot S}{P \cdot S D_{i}}\right) \quad i \in\left[1, N_{C}\right]
$$

where $l_{i}$ indicates the number of leaf nodes depending of the $i$-th coordinator, $n_{B I}$ is the number of packets per Beacon Interval emitted by a leaf node, $S$ is the packet size (in bits) including all headers, $P$ is Zigbee rate (250 Kbps) and $S D_{\mathrm{i}}$ is the Superframe Duration of the $i$-th cluster.

In the tested networks we vary the traffic conditions by modifying $n_{B I}$ (which is a positive integer). The presented results correspond to simulations with heavy traffic conditions for which $\rho$ was obviously designed to be less than $100 \%$. In all the scenarios, for values of $\rho$ above $60 \%$, CSMA/CA collisions in most overloaded clusters provoke strong loss rates and instabilities in the network so that results could not be considered representative (see [9] for a detailed analysis of the behavior of CSMA/CA in 802.15.4 networks) .

As the metric to characterize the network performance, we utilize the achieved throughput defined as the mean bit rate (per leaf node) at which the ZC actually receive the data from each leaf node (without taking into consideration the packets that get lost or have to be retransmitted because of a collision). In particular the throughput is calculated as the ratio between the number of bytes 
that are successfully transmitted per leaf node and per superframe and the Beacon Interval.

Table 1 summarizes other parameters utilized for the simulations.

The results (achieved throughput and designed values for the Superframe Orders of the coordinators) for the three analyzed topologies are included in Tables 2, 3 and 4. The tables show that in all cases, for similar values of $\rho$, the topology based distribution noticeably outperforms the other two basic algorithms. For the scenarios 1 and 2 where several coordinators receive the traffic from the leaf nodes, the adaptive algorithm yields higher values for all the Superframe Orders. This means that the algorithm is able to maximize the utilization of the Beacon Interval so that the periods in which no cluster is active are minimized. This optimized design of the activity periods permits to improve the network throughput substantially (see [10] for a thorough study of the impact of the Superframe Order on data rate in Zigbee star topology networks)

As it could has been expected, the results of the topologies in which the Zigbee coordinator concentrates the traffic from several clusters (especially the scenario 2) evidence that resources cannot be equally distributed among the clusters. Thus, the superframe duration of the Zigbee Coordinator should be higher than the time reserved for the other clusters. Otherwise, the ZC becomes an important bottleneck for the network. But, similarly, if the Superframe Order of the ZC is blindly privileged without considering the load in the Zigbee Routers, this parameter could be unnecessarily overdimensioned at the cost of reducing the resources of the ZRs and transferring the bottleneck to another lower level cluster in the network. Remark, for example, that even in the second scenario (where the ZC must support the traffic from 4 coordinator) the fixed prioritization of the ZC just matches the results obtained by the first algorithm while in the first scenario (where the clusters to multiplex are just two) the achieved throughput is quite lower.

On the other hand, the scenario 3 shows the limit case in which a cluster coordinator has to transport the same traffic of the Zigbee Coordinator. In that case, the SO order of both clusters must be necessarily the same. Results show that the topology based algorithm is capable of adjusting this situation by assigning the same values for the Superframe Orders of both the Zigbee Coordinator and Routers. In contrast, a very poor performance is obtained if the ZC is prioritized in this scenario (as far as a strong bottleneck is originated in the lower level router).

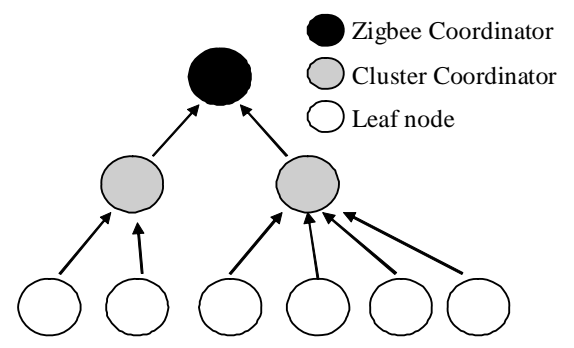

Figure 5. Scenario 1

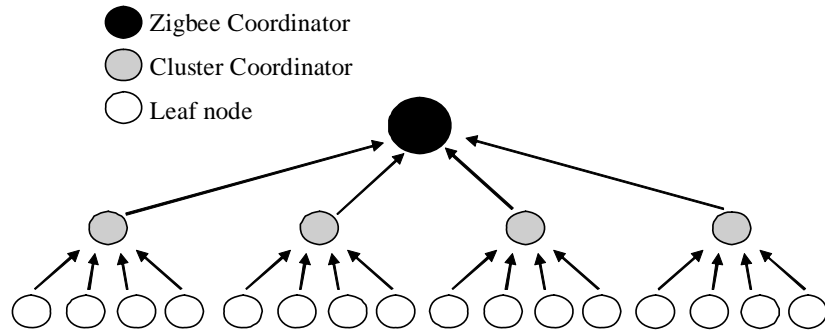

Figure 6. Scenario 2

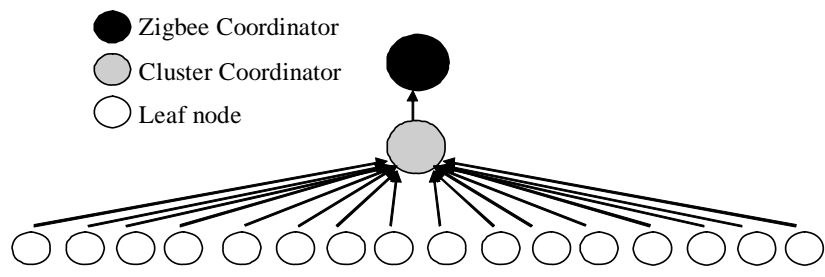

Figure 7. Scenario 3

Table 1. Other parameters of the simulation

\begin{tabular}{|c|c|}
\hline Parameter & Value \\
\hline $\begin{array}{l}\text { Beacon Order }(B O) \\
\text { (Beacon Interval, } B I)\end{array}$ & $\begin{array}{c}8 \\
(3.932 \mathrm{~s})\end{array}$ \\
\hline Contention Window (CW) & 0 slots \\
\hline $\begin{array}{l}\text { Packet size(including the 6-byte Physical layer } \\
\text { header and the } 15-\text {-to- } n \text { byte MAC sublayer header) }\end{array}$ & 108 bytes \\
\hline $\begin{array}{c}\text { No of packet retransmissions in case of collision } \\
\text { (CSMA/CA algorithm) }\end{array}$ & 7 \\
\hline
\end{tabular}

Table 2. Designed values of Superframe Orders and achieved throughput per leaf node (scenario 1)

\begin{tabular}{|c|c|c|c|c|c|}
\hline \multicolumn{5}{|c|}{ Policy for determining the Superframe Orders } \\
\hline $\begin{array}{c}\text { Same SO for all } \\
\text { nodes }\end{array}$ & $\begin{array}{c}\text { Prioritization of } \\
\text { Zigbee } \\
\text { coordinator (ZC) }\end{array}$ & $\begin{array}{c}\text { Topology Based } \\
\text { Distribution }\end{array}$ \\
\hline \multicolumn{2}{|c|}{$S O=6$} & \multicolumn{2}{|c|}{$\begin{array}{c}S O_{1}=6 \\
S O_{2,3}=3\end{array}$} & \multicolumn{2}{|c|}{$\begin{array}{c}\mathrm{SO}_{1}=7 \\
\mathrm{SO}\end{array}$} \\
\hline $\boldsymbol{\rho}$ & Throughput & $\boldsymbol{\rho}$ & Throughput & $\boldsymbol{\rho}$ & Throughput \\
\hline $\mathbf{5 5 \%}$ & 4210 bps & $\mathbf{5 6 \%}$ & 810 bps & $\mathbf{5 6 \%}$ & 6478 bps \\
\hline $\mathbf{4 7 \%}$ & 3562 bps & $\mathbf{4 5 \%}$ & 648 bps & $\mathbf{4 6 \%}$ & 5371 bps \\
\hline $\mathbf{4 0 \%}$ & 3077 bps & $\mathbf{3 4 \%}$ & 486 bps & $\mathbf{4 1 \%}$ & 4720 bps \\
\hline
\end{tabular}


Table 3. Designed values of Superframe Orders and achieved throughput per leaf node (scenario 2)

\begin{tabular}{|c|c|c|c|c|c|}
\hline \multicolumn{5}{|c|}{ Policy for determining the Superframe Orders } \\
\hline $\begin{array}{c}\text { Same SO for all } \\
\text { nodes }\end{array}$ & $\begin{array}{c}\text { Prioritization of } \\
\text { Zigbee } \\
\text { coordinator (ZC) }\end{array}$ & $\begin{array}{c}\text { Topology Based } \\
\text { Distribution }\end{array}$ \\
\hline \multicolumn{2}{|c|}{ SO=5 } & \multicolumn{2}{|c|}{$\begin{array}{c}\mathrm{SO}_{1}=6 \\
\mathrm{SO}_{2,3,4,5}=3\end{array}$} & \multicolumn{2}{|c|}{$\begin{array}{c}\mathrm{SO}_{1}=7 \\
\mathrm{SO}_{2,3,4}=5\end{array}$} \\
\hline $\boldsymbol{\rho}$ & Throughput & $\boldsymbol{\rho}$ & Throughput & $\boldsymbol{\rho}$ & Throughput \\
\hline $\mathbf{5 6 \%}$ & 814 bps & $\mathbf{5 6 \%}$ & 810 bps & $\mathbf{5 6 \%}$ & 3255 bps \\
\hline $\mathbf{4 5 \%}$ & 651 bps & $\mathbf{4 5 \%}$ & 648 bps & $\mathbf{4 5 \%}$ & 2604 bps \\
\hline $\mathbf{3 4 \%}$ & 488 bps & $\mathbf{3 4 \%}$ & 486 bps & $\mathbf{3 9 \%}$ & 2278 bps \\
\hline
\end{tabular}

Table 4. Designed values of Superframe Orders and achieved throughput per leaf node (scenario 3)

\begin{tabular}{|c|c|c|c|c|c|}
\hline \multicolumn{6}{|c|}{ Policy for determining the Superframe Orders } \\
\hline \multicolumn{2}{|c|}{$\begin{array}{l}\text { Same SO for all } \\
\text { nodes }\end{array}$} & \multicolumn{2}{|c|}{$\begin{array}{c}\text { Prioritization of } \\
\text { Zigbee } \\
\text { coordinator (ZC) }\end{array}$} & \multicolumn{2}{|c|}{$\begin{array}{l}\text { Topology Based } \\
\text { Distribution }\end{array}$} \\
\hline \multirow{2}{*}{\multicolumn{2}{|c|}{$S O=7$}} & \multirow{2}{*}{\multicolumn{2}{|c|}{$\begin{array}{l}S O_{1}=6 \\
S O_{2}=3\end{array}$}} & \multirow{2}{*}{\multicolumn{2}{|c|}{$\begin{array}{l}\mathrm{SO}_{1}=7 \\
\mathrm{SO}_{2}=7\end{array}$}} \\
\hline & & & & & \\
\hline$\rho$ & Throughput & $\rho$ & Throughput & $\rho$ & Throughput \\
\hline $39 \%$ & 2604 bps & \multirow{3}{*}{$45 \%$} & \multirow{3}{*}{163 bps* } & $39 \%$ & 2604 bps \\
\hline $36 \%$ & 2115 bps & & & $36 \%$ & 2115 bps \\
\hline $34 \%$ & 1953 bps & & & $34 \%$ & 1953 bps \\
\hline
\end{tabular}

*Note: for the second evaluated algorithm higher traffic loads $(\boldsymbol{\rho})$ provoke strong instabilities in the lower level cluster. The load $\rho=45 \%$ corresponds with the emission of just one packet per superframe. Lower loads cannot be simulated with the utilized traffic pattern.

\section{CONCLUSIONS AND FUTURE WORK}

This article has investigated the problem of configuring hierarchical 802-15.4/Zigbee cluster-trees. In order to avoid beacon collision, the study has focused on several strategies to distribute the Beacon Interval among the clusters to guarantee that no beacon collisions occur.

Simulations show that even in small networks with less than twenty nodes a proper design of the duration of the 802.15.4 superframes is a key aspect to achieve a reasonable network performance. Results demonstrate that the policies that distribute the beacon interval in a rough way without taking into account the topology and traffic condition in the PAN most probably will lead to an inefficient network design with a extremely limited actual data throughput.
The paper has proposed an iterative algorithm that tries to optimize the utilization of the network Beacon Interval by adjusting the superframe duration of the clusters to the traffic supported by the coordinators. Future work should investigate the adaptation of this type of algorithms to more complex situations when, for example, node mobility is allowed and topology dynamically changes, when all coordinators are not assumed to be neighbors or when the Beacon Interval of each cluster is different from the rest.

\section{ACKNOWLEDGMENTS}

This work was partially supported with public funds by the Spanish National Project No.TEC2006-12211-C02-01.

\section{REFERENCES}

[1] IEEE-TG15.4, "Part 15.4: Wireless Medium Access Control (MAC) and Physical Layer (PHY) Specifications for LowRate Wireless Personal Area Networks (LR-WPANs)", IEEE standard for Information Technology, 2003.

[2] Zigbee-Alliance, "ZigBee specification", URL: http://www.zigbee.org/, 2005.

[3] Hardware platforms available for TinyOS, described at: http://www.tinyos.net/

[4] IEEE 802.15 WPANTM Task Group 4b, URL: http://grouper.ieee.org/groups/802/15/pub/TG4b.html

[5] Koubâa, A., Alves, M., Attia, M. and Van Nieuwenhuyse, A., "Collision-Free Beacon Scheduling Mechanisms for IEEE 802.15.4/Zigbee Cluster-Tree Wireless Sensor Networks", in Proceedings of the Workshop for Applications and Services in Wireless Networks, July 2007.

[6] Neugebauer, M., Plönnigs, J. and Kabitzsch, K., “A New Beacon Order Adaptation Algorithm for IEEE 802.15.4 Networks", In Proceedings of the 2nd European Workshop on Wireless Sensor Networks (EWSN 2005), Istanbul (Turkey), January 2005, pp. 302-311.

[7] Koubâa, A., Cunha, A. and Alves, M., "A Time Division Beacon Scheduling Mechanism for IEEE 802.15.4/Zigbee Cluster-Tree Wireless Sensor Networks", In Proceedings of 19th Euromicro Conference on Real-Time Systems (ECRTS 2007), Pisa (Italy), July 2007.

[8] Chipcon, URL: http://www.chipcon.com/

[9] Lee, T.J., Lee, H.R. and Chung M.Y., "MAC Throughput Limit Analysis of Slotted CSMA/CA in IEEE 802.15.4 WPAN”, IEEE Communications Letters, Vol. 10, no. 7, July, 2006, pp. 561-563.

[10] Ko, L., Liu, Y. and Fang, H., "Design and implementation of IEEE 802.15.4 beacon-enabled network devices", In Proceedings of the Fourth Annual IEEE International Conference on Pervasive Computing and Communications Workshops (PERCOMW'06), Pisa (Italy), March 2006, pp. 415. 